\title{
Enabling Multi-Material 3D Printing for Designing and Rapid Prototyping of Deformable and Interactive Wearables
}

\author{
Aluna Everitt \\ aluna.everitt@cs.ox.ac.uk \\ University of Oxford \\ Oxford, United Kingdom
}

\author{
Alexander Keith Eady \\ alexeady@cmail.carleton.ca \\ Carleton University \\ Ottawa, Ontario, Canada
}

\author{
Audrey Girouard \\ audrey.girouard@carleton.ca \\ Carleton University \\ Ottawa, Ontario, Canada
}
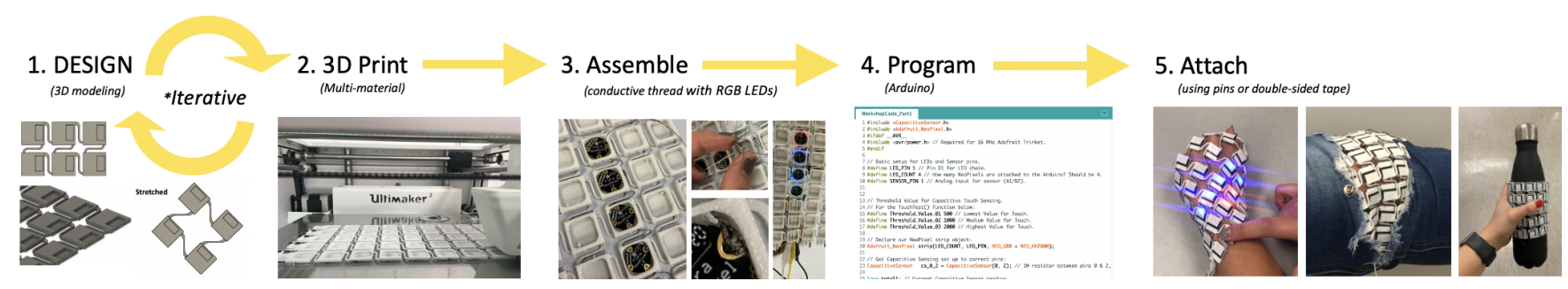

Figure 1: The workflow pipeline for our design and fabrication approach follows 5 steps. In step 1, users first use software to design the deformable surface. For step 2, they then 3D print the surface with an off-the-shelf multi-material 3D printer. During step 3, they then assemble the electronics using conductive thread and in step 4 they program the functionality. Step 5 demonstrates our deformable surface attached to the body, integrated onto a garment, and secured to another mobile object.

\begin{abstract}
Deformable surfaces with interactive capabilities provide opportunities for new mobile interfaces such as wearables. Yet current fabrication and prototyping techniques for deformable surfaces, that are both flexible and stretchable, are still limited by complex structural design and mechanical surface rigidity. We propose a simplified rapid fabrication technique that utilizes multi-material 3D printing for developing customizable and stretchable surfaces for mobile wearables with interactive capabilities embedded during the 3D printing process. Our prototype, FlexiWear, is a dynamic surface with embedded electronic components that can adapt to mobile body shape/movement and applied to contexts such as healthcare and sports wearables. We describe our design and fabrication approach using a commercial desktop 3D printer, the interaction techniques supported, and possible application scenarios for wearables and deformable mobile interfaces. Our approach aims to support rapid development and exploration of deformable surfaces that can adapt to body shape/movement.
\end{abstract}

\section{CCS CONCEPTS}

- Human-centered computing $\rightarrow$ User interface toolkits.

\footnotetext{
Permission to make digital or hard copies of all or part of this work for personal or classroom use is granted without fee provided that copies are not made or distributed for profit or commercial advantage and that copies bear this notice and the full citation on the first page. Copyrights for components of this work owned by others than the author(s) must be honored. Abstracting with credit is permitted. To copy otherwise, or republish, to post on servers or to redistribute to lists, requires prior specific permission and/or a fee. Request permissions from permissions@acm.org.

MUM 2021, December 5-8, 2021, Leuven, Belgium

(c) 2021 Copyright held by the owner/author(s). Publication rights licensed to ACM. ACM ISBN 978-1-4503-8643-2/21/05 ...\$15.00

https://doi.org/10.1145/3490632.3490635
}

\section{KEYWORDS}

Fabrication, Prototyping, 3D Printing, Wearables, Mobile Interface Design

\section{ACM Reference Format:}

Aluna Everitt, Alexander Keith Eady, and Audrey Girouard. 2021. Enabling Multi-Material 3D Printing for Designing and Rapid Prototyping of Deformable and Interactive Wearables. In 20th International Conference on Mobile and Ubiquitous Multimedia (MUM 2021), December 5-8, 2021, Leuven, Belgium. ACM, New York, NY, USA, 11 pages. https://doi.org/10.1145/ 3490632.3490635

\section{INTRODUCTION}

There is growing interest for fabricating deformable devices with stretchable surfaces that can adapt to the body and movement $[47,52]$. Research on these devices is diverse and explores form, interaction, sensing capabilities, visualization $[11,56]$, as well as covering a broad set of applications [52]. Applications range across generalized topics such as health monitoring to more specific areas such as firefighting [19]. With a wide range of applications come design challenges for conceptualizing, developing, and testing devices that can be easily worn on the body, follow movements, and/or attach to existing garments. As the domain of wearables matures and expands, we see the need to further support the design and development of wearable technologies, particularly with rapid prototyping approaches [40].

Current fabrication techniques for developing deformable wearables are often limited by thickness of electronics and mechanical surface rigidity [38]. Ultra-thin on-body devices provide more flexibility but often contain chemical compounds for printing with conducting polymer that may cause irritation on direct contact with sensitive skin (e.g. PEDOT:PSS/Gwent C2100629D1) [82]. Etextiles are commonly used for interactive deformable wearables 
and mobile devices [31, 34], though they are often limited in supporting rapid fabrication and customization of material properties, e.g. stretchability/elasticity and bendability on demand and/or at specific locations [47]. Much like e-textiles require specialized machinery $[48,57]$ (e.g. knitting machines), FDM 3D printers can also be used to prototype interactive wearables with more complex geometries and configurations [51]. Alternative methods of prototyping wearables require more advanced materials such as silicone [53] and Polydimethylsiloxane [84] that come at high cost and complexity (e.g. multi-layered approaches require more assembly).

The need for rapid prototyping mobile interfaces is acknowledged within the field of Human-Computer Interaction (HCI). For example, Leber and Madrid [40] present a platform for rapidly prototyping wearables that can be used to augment the body with an array of sensors. However, it is limited to placing sensors only on an existing garment (e.g. a vest). When designing wearables, a multitude of forms and applications that can be explored and proposed, particularly because these devices can be situated on both on the body and on a garment. We see the need for a prototyping approach that is adaptable and can support the design of wearables both directly on-body or within a garment as well as support embedded sensing and interaction capabilities. We also emphasis the need for context dependent wearables which provide device functionality based on the context of use [14]. To achieve this, we highlight the need to support rapid and accessible fabrication approaches. Additionally, research [43] indicates a need for rapid prototyping methods to expand the design space for fabricating deformable and interactive mobile devices.

Our core motivation is to provide the mobile research and design communities with an alternate method to rapidly fabricating deformable wearable and mobile prototypes that can be worn on skin (Figure 2A), integrated in garments (Figure 2C), or attached to portable objects (Figure 1). We demonstrate this by presenting a rapid and customizable fabrication approach that can support a range of forms, is easily augmented with off-the shelf components, and is mobile. By utilising Computer Aided Design (CAD), we support fast iterations (print times and modifications) and inexpensive and easily sourced materials, which speed up assembly with commonly available tools.

We demonstrate how multi-material 3D printing can be utilised as a rapid prototyping method (Figure 1) for developing wearables with our proof of concept prototype (Figure 2). We employ multimaterial Fused Deposition Modeling (FDM) 3D printing where: (1) Flexible filament provides deformable and stretchable surfaces and, simultaneously. (2) Conductive filament is embedded to create interactive areas, through capacitive touch circuits, within the stretchable surfaces all in a single print. The surface design includes gaps and channels that, post-printing, accommodate Light-Emitting Diodes (LEDs) to support output visualization (e.g. for at a glance feedback) together with capacitive sensing capabilities. See Figure 2D for details. The structural design of our device prototype, FlexiWear, allows it to deform and follow the form of the wearer's body during movement as it can be worn on-body or attached to a garment (Figure 2A-C). Please see Supplementary Material for the STL 3D models of our prototype for 3D printing.

Our main contribution is a design and fabrication approach (Figure 1) for developing deformable wearables with integrated interaction and visualisation capabilities realised through multimaterial 3D printing. By integrating flexible and conductive materials simultaneously during printing, we rapidly create customizable interactive wearable surfaces, at low-cost, where the tile design enables the surface to fit around body parts. Our proof of concept prototype (Figure 2), a thin and stretchable wearable surface that easily adapts to on-body or in-garment movement and deformations. Our approach and resulting prototype demonstrates how multi-material 3D printing together with the specified structural surface design offers rapid fabrication of deformable and interactive wearables.

\section{RELATED WORK}

We explore current work related to fabrication approaches for wearables, deformable user interfaces, and how they support the development of our design and fabrication approach as well as final prototype.

\subsection{Fabrication Approaches for Wearables and Deformable Surfaces}

Fabrication approaches for rigid wearables (e.g. commercial smartwatches) follow well established electronic and fabrication prototyping methods $[35,44,53]$. Below, we detail the fabrication approaches for wearables commonly adopted for deformable surfaces. We also discuss general fabrication methods that include electronic prototyping within 3D printing.

2.1.1 Fabricating Stretchable Surfaces. With the increase in personal fabrication [8] 3D printing offers a more widely accessible approach for developing wearables as they are commonly found within maker-spaces and even some households. Rivera et al. [66] illustrate how 3D printing and textiles complement, with textileembedded 3D printed objects that are flexible and stretchable. Wong and Hernandez [85] review current additive manufacturing processes for 3D printing. Schumacher et al. [71] present a method to characterize the mechanical behavior of structured sheet materials allowing users to capture deformational behavior under stretching and bending. With their approach users can explore various structures and design/create materials with specific desired micro mechanical properties. Ultimately, their work allows people to define and fabricate structures with a variety of desired material properties, particularly for bending and stretching. Silicon based fabrication techniques can also be used to develop deformable wearables [13].

Laser cut solid segments can also be attached to stretchable Spandex to fabricate flexible surfaces, specifically for producing shape-changing applications [16]. Similarly, LASEC [23] is another example of a fabrication technique that utilizes the rapid nature of laser cutters for developing deformable circuits with custom stretch-ability. Their prototypes enable both stretchable and transparent wearable devices to be rapidly fabricated using a single layer of conductive material. By utilizing multi-material 3D printing our approach provides design freedom in 3D space and more customization options for post-print components that can speed fabrication. 
2.1.2 E-Textiles for Wearables. E-textiles comprise electronic components directly incorporated with fabrics to create interactive surfaces $[12,33,75]$. This includes recent work that explores touchless gestural input with interactive fabrics [86]. To build interactive surfaces for wearables with the fluidity of textiles and interactive features, e-textiles employ conductive yarns, optical fibers, printing with conductive ink, and connecting electronic components with microcontrollers [10, 28, 42, 46, 61]. While these approaches can be used for wearables and mobile devices [31, 34], they pose several fabrication limitations. Fabrication requires extensive manual assembly and electronic components can be exposed to the wearer or others nearby [20]. Woven optical fibers [26] can create cloth-like materials that act as flexible displays without exposing wiring and electronics to users, however, this technique requires an external infrared signal input device (e.g. laser pen). FunCushion [30] introduces a method for discretely integrating electronics within textiles. Sugiura's et al. [74] pressure sensing method uses infrared sensors hidden within household soft objects for interaction and integrated lights for visualization to create 3D cloth displays.

Digital fabrication methods for deformable textile surfaces also enable end-users and designers to create interactive fabric-like applications $[29,54,60]$. However, these techniques do not yet have established rapid fabrication processes that are reproducible without a high level of technical skill. Sketch\&Stitch [25] begins to establish a DIY interactive design process for rapidly fabricating e-textiles by mapping user's drawings directly to computer-controlled embroidery machines. Though this system removes barriers to users with lower technical skill, this approach still requires specialized machines (e.g. computer-controlled embroidery machines) unlike 3D printers that provide more generalized functionality in comparison $[8,50]$. Similar to computer-controlled embroidery machine use for e-textiles, McCann et al. [48] demonstrate how computer-controlled knitting machines utilize automatic assemblies of high-level shape primitives (tubes, sheets) into low-level machine instructions to construct knitted objects. Though this approach is promising for minimizing assembly, the user is limited to yarn materials and are limited to specific automated knitting machines. More conventional fabrication technologies, such as 3D printers, are more commonly available in maker spaces and libraries.

\subsection{D Printing with Embedded Electronics}

Printed flexible circuits are increasingly used for a range of applications as they create bend or touch sensors [22], typically made using inkjet printing. They often require additional assembly, e.g. attached to $3 \mathrm{D}$ objects or within enclosures [74]. Lee et al. [41] provide a review on $3 \mathrm{D}$ printed smart devices for $4 \mathrm{D}$ printing. Particularly, they detail recent work on multi-material 3D printing with electrically functional materials including conducting, sensing, insulating and semiconducting materials that has led to the development of smart devices such as 3D structural electronics, sensors, batteries, etc. In the field of soft robotics 3D printing has also been utilized to create pneumatic actuators with embedded sensing [79]. Our use of multi-material 3D printing reduces assembly time, with the core device fabricated in one print. Schmitz et al. [69] present a fabrication pipeline to design and 3D print capacitive touch sensors for interactive objects. EVA Moccasin [51] also uses a 3D printing approach in creating wearables, specifically for prototyping smart shoe soles. We follow similar approaches, creating capacitive touch sensors with multi-material 3D printing.

Savage et al. [68] describe the design and development of interactive devices by embedding optical light tubes within 3D printed objects. A subtractive algorithmic approach generates space within 3D models to accommodate post-print manual insertion of components like sensors or actuators for interaction. Our focus is similar, preparing the surfaces during the design phase to include sensors (i.e. using conductive filament) or to enable electronics to be embedded after with minimal assembly [49] (e.g. at most three steps).

\subsection{Interaction with Context Dependent Wearables}

Context dependent wearables provide device functionality based on the context of use [14]. Work in this are illustrates several important design requirements for closely worn devices. Wearables must (1) adapt to and with the body's movement, and (2) support the organic and dynamic shape of the human body alongside our changing needs in different settings. Snaplet [78] provides context dependent functionality with sensors embedded in a flexible display to detect deformation. Similarly, Facet [45], a multifaceted digital bracelet, reconfigures to match user needs.

Wearables can also adapt to and support interactions that occur off the device and even off the body. Doppio [73], a reconfigurable smartwatch, allows one of its two touch sensitive displays to be detached and shared with others. SleeD [81] is a sleeve-like wearable that supports interaction with touch-sensitive walls, other devices, and people. Context aware wearables can also adapt to dynamic social factors. AugmentedForearm [56], a sleeve worn wearable with multiple displays, transitions between public and private states based on arm orientation and direct user interaction. This work highlights the importance of designing wearables that acknowledge how the public and personal aspects of clothing can overlap. Our approach supports the design of context aware and adaptable wearables. The conductive filament used during printing, creates integrated capacitive sensors within the deformable surface with no additional assembly required. By monitoring capacitance and establishing thresholds we can detect whether it is worn on or off the body, along with changes in its shape, or additional touching.

\subsection{Flexible and Stretchable Interfaces and Skins}

Deformable User Interfaces (DUIs) can be deformed as a means of interaction through physical actions like squeezing, bending, and stretching [36]. They support a range of tangible interactions and are diverse in their degree of flexibility and malleability [17, $72,80,84]$. Jensen et al. [32] present TransPrint, a method which offers a way of creating bendable interactive displays using as electrochromic manufacturing technique. Prototypes developed from these methods can adapt to fit dynamic organic shapes like the human body - making them ideal for wearable devices $[58,78]$.

Current wearables commonly employ flexible touch-screens [11] or LEDs embedded in a rigid enclosure (e.g. FitBit). Olberding et al. [56] highlight that wearable displays must be stretchable to ensure the device does not impede human motor systems when the user 
is turning and bending for instance. Non-stretchable wearables limit the rotation and twisting capabilities of the user's body parts. Transparent and flexible woven electrodes [39] and smart fabric technologies [59] are a promising direction, though commercially they are yet to be adopted for stretchable interfaces.

Work on thin skin overlays supports thin and stretchable wearable input devices [82] and tattoo-based approaches [35, 44, 55, 83], though these approaches have yet to go beyond prototyping wearables. Thin skin overlay wearables, such as SkinMarks [83], can supports display output, deformation sensors, and context/bodypose dependent input controls though often require chemical compounds for printing and masking with conducting polymers. These processes are often time consuming and the chemical compounds involved (e.g. PEDOT:PSS conducting polymer) may not be suitable for prolonged direct contact on skin. To support a more accessible and rapid fabrication approach we focus on more commercially available techniques for fabrication (e.g. 3D printing) without the need for harsh chemical use directly on skin.

Some wearables use tiled displays loosely connected and directly attached to the skin, to ensure the human motor system and movement remain unaffected [56], though this limits the resolution and scale of visualizations. Wearable displays also use flexible electrophoretic displays [65], arrays of large tiles [64] where multiple screens are attached in a row $[45,56]$, or augmented e-textiles [34].

Functional nano-materials for wearables are a promising direction for slim devices that seamlessly adapt to human body and skin. However, recent works highlight that high cost and lack of accessibility limit these fabrication processes [38, 63]. FlexiWear utilizes $3 \mathrm{D}$ printing and $\mathrm{CAD}$ for developing flexible and stretchable wearables that meet key interaction principles for DUIs. Our fabrication approach reflects the vision of Olberding et al. [56] for flexible devices, demonstrated by FlexiWear, whose stretchable properties support use on body parts, such as the knee, which undergo a wide range of motion.

\section{DESIGN AND FABRICATION APPROACH}

The main premise of our fabrication approach is to use multimaterial 3D printing to rapidly create flexible surfaces that have embedded interactivity, are deformable, and can be worn by a user. Figure 1 shows the end-to-end workflow of our design and fabrication approach which consists of 5 steps, where steps 1 and 2 are iterative. To demonstrate the versatility of multi-material 3D printing for prototyping we developed FlexiWear, a prototype device that can be worn on-body or attached to a garments (Figure 2A-C). The design comprises of tiles (sizes $10-15 \mathrm{~mm}$ ) that are linked by thin s-shaped sections, which allow the device to bend and stretch easily. Below we provide an overview of our workflow pipeline together with details for 3D modeling design rational and materials required to replicate the wearable prototype.

Figure 1 shows the workflow of our design and fabrication approach where for step 1, we use Computer Aided Design (CAD) software for 3D modeling a custom deformable surfaces with interactive sections and areas for embedding electronics. In step 2, multi-material FDM 3D printing with flexible filament is used to produce the deformable surfaces that also support capacitive touch sensing capabilities using conductive filament. Steps 1 and 2 are part

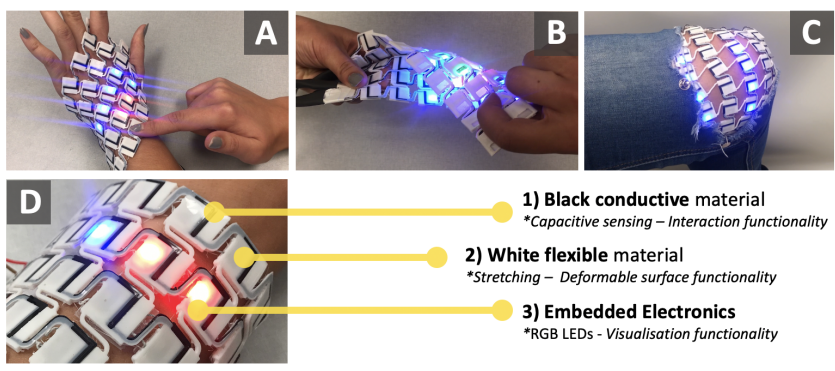

Figure 2: Our 3D printed prototype with capacitive touch sensing and integrated LED electronics. Demonstrating interaction techniques for pressing (A), bending (B), and stretching $(C)$ on the hand and integrated into a pair of jeans. Breakdown of prototype elements (D).

of an iterative process where the design 3D model can be readjusted after printing test prototypes. For step 3, we assemble the device by placing electronic components into designated cavities in the $3 \mathrm{D}$ printed surface and then 'sewing/threading' together the circuit using conductive thread. During step 4, a small micro-controller such as an Arduino Nano or the wearable Adafruit Gemma is used with Arduino IDE to program the electronics. Finally for step 5, once the surface is fully assembled and functioning it can be attached either to the body using double sided body tape [77], attached to clothing using pins, or other objects using conventional double sided tape or super glue.

\subsection{Step 1: Design of Deformable Surface}

We use Fusion360 to model tile and link designs. With each tile linked, the print behaves as a continuous flexible surface. Figure 3 shows our 3D model design example. The tile dimensions $(15 \times 15 \times 2$ $\mathrm{mm}$ ) can accommodate miniature electronics (e.g. surface mount LEDs) and are linked together during the printing process where, in aggregate, they form a flexible and lightweight interactive prototype device. A gap of $5 \mathrm{~mm}$ between tiles, where a curved link connects each tile, produces a stretchable surface. For interaction, a layer of conductive filament ( $0.5 \mathrm{~mm}$ depth) is embedded during printing on each tile and is connected through the links (Figure $3 \mathrm{~A}$ ).

We use NeoPixel LEDs $[2,3]$ for their miniature size and compatibility with wearable applications. A space ranging between $5 \times 10 \times 1.5 \mathrm{~mm}(\mathrm{~min})$ to $12.75 \times 12.75 \times 2.80 \mathrm{~mm}(\max )$ in each tile can be used to set an LED. We used white flexible 3D printing material [18], which also served to diffuse light from the LEDs throughout each tile, making them more like large pixels in a display. The number of tiles and LEDs reflects the resolution of the wearable. With more tiles, more complex visualizations can be created. In our design of the FlexiWear prototype, we use between two to three gaps (1x1 $\mathrm{mm}$ each) in every tile that allow conductive thread or insulated electromagnetic wire $(0.5 \mathrm{~mm})$ to be threaded through the prototype and provide current and data for the LED chain (Figure 3B). We daisy chain the electronics in sequence (Figure 7) to address individual components. Below, we describe the design iterations of the tiles and links and how their geometry affects bendability, stretching, and conductivity. 

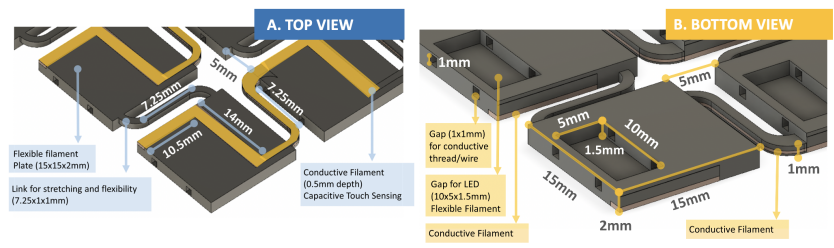

Figure 3: Prototype tile and link 3D model design close-up that is merged in aggregate to create a FlexiWear device.

3.1.1 Tile Design. First, we explored the impact of varying tile and link dimensions and shapes to support surface deformation (Figure 4), which is affected by the arrangement of the tiles and the links between them. Tiles in a triangular configuration (Figure 4B) allow 60-degree bends on an angle parallel to the triangle sides. This limits deformation to provide a flexible, but not cloth-like, surface. For our final prototype, we use square tiles as they enable a 90-degree bend in four directions without any obstructions. We limit the tile depth to $2 \mathrm{~mm}$, leaving the overall prototype lightweight. This also reduces printing time and material required for fabrication.

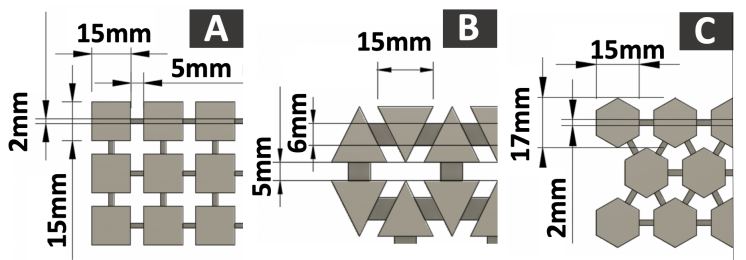

Figure 4: Initial tile design explorations with square (A), triangular (B), and hexagonal (C) polygons.

3.1.2 Link Design. Secondly, we explore links that allow stretching of 3D printed surfaces. Inspired by 3D printed spring mechanisms [27], our stretchable links use flexible filament which has elastic properties. After deformation, tiles regain their original shape like most auxetic style materials [15]. The stretch factor between tiles is facilitated by the "S" shape and link length. This link connection design creates an anisotropic flexibility with limited stretching. This means that the stretching ability is limited along the direction of the power lines (Figure 5D). To overcome this limitation more power lines can be added each link, much like a spring has more coils to increase the length of stretch.

Deformation of the 3D printed surface is affected by width and thickness of the link. In our initial link design, we used straight lines to connect each tile to understand how width and depth of the flexible material affects bending in aggregate. We printed link widths of $0.5 \mathrm{~mm}, 1 \mathrm{~mm}$, and $2 \mathrm{~mm}$. We found that $1 \mathrm{~mm}$ link was more durable than the $0.5 \mathrm{~mm}$ link and also more stretchable than the $2 \mathrm{~mm}$ link. Our original link designs were $2 \mathrm{~mm}$ wide (Figure $4 \mathrm{~A} / \mathrm{C}$ ), from our design iterations we recommend link width of 1 $\mathrm{mm}$ and depth of $1 \mathrm{~mm}$ for maximum flexibility and durability. A wider link (Figure 5A) reduces bending, resulting in a more rigid form. Thinner links (Figure 5B) bend easily under less force. Our final link design (Figure 5C), uses a "S" shape that allows horizontal stretch (Figure 5D).

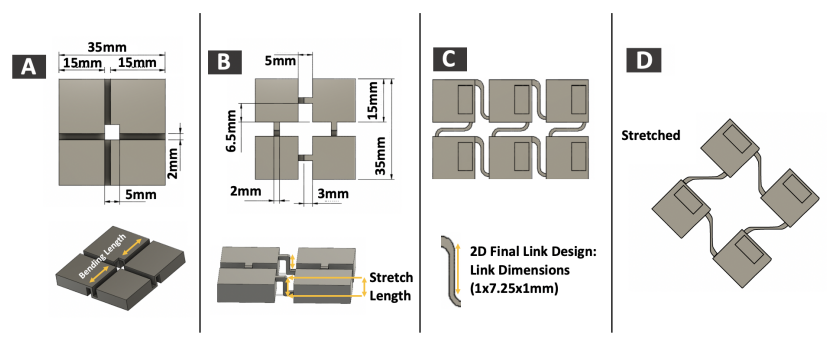

Figure 5: Link design explorations; wider vertical link design (A), thinner vertical link (B), final horizontal curved link (C), curved link stretched (D).

Table 1: List of printing parameters with their respective values used to fabricate our prototype on the Ultimaker 3.

\begin{tabular}{l|l|l}
\hline Parameter & $\begin{array}{l}\text { Flexible Filament } \\
\text { (value) }\end{array}$ & $\begin{array}{l}\text { Conductive Filament } \\
\text { (value) }\end{array}$ \\
\hline Print Spree (mm/s) & 30 & 30 \\
Print Temperature & 215 & 215 \\
Build Plate Temperature & 40 & 40 \\
Fan Speed (\%) & 20 & 100 \\
Build Plate Adhesion & None & None \\
\hline
\end{tabular}

\subsection{Step 2: 3D Printing, Materials, and Electronic Components}

We utilize multi-material 3D printing using the Ultimaker 3 dual extrusion FDM 3D printer. Table 1 provides a breakdown of parameter values for each material. For the flexible material we use commercially available Cheetah Snow White 95A TPU [18] (3mm diameter, $1 \mathrm{~kg}$ at $\$ 85.00$ per spool) for fabricating a $2 \mathrm{D}$ array of tiles that are linked together to create a stretchable surface (Figure 2D). Each spool of flexible filament makes 15 surfaces (length $=185 \mathrm{~mm}$, width $=95 \mathrm{~mm}$, depth $=3.9 \mathrm{~mm}$ ) where cost per unit is $\$ 5.67$. For the conductive material we use Protopasta Electrically Conductive Composite PLA [62] (2.85mm diameter, 500g at $\$ 49.99$ per spool) to print capacitive touch sensors within the flexible surfaces [70]. Approximately half a spool is used to print 24 surfaces. Though conductive PLA is not a flexible filament, when applied as a single layer $(0.2 \mathrm{~mm})$ and encased in flexible material, it can behave as such. Gaps in the tiles allow for LEDs to be inserted within the surface and channels for wiring and conductive thread (Figure 3B) allow us to embed electronics.

In terms of electronic components, for the micro-controller we use either an Arduino Nano [6] or a wearable Adafruit Gemma v2 [1]. For visualisation, we use either single colour Adafruit LED Sequins [2] or more advanced colour programmable Adafruit Flora RGB Smart NeoPixel v2 [3]. We calculate the full cost of fabricating one prototype can ranges between $\$ 20-\$ 34$, particularly depending on the choice of micro-controller used. 

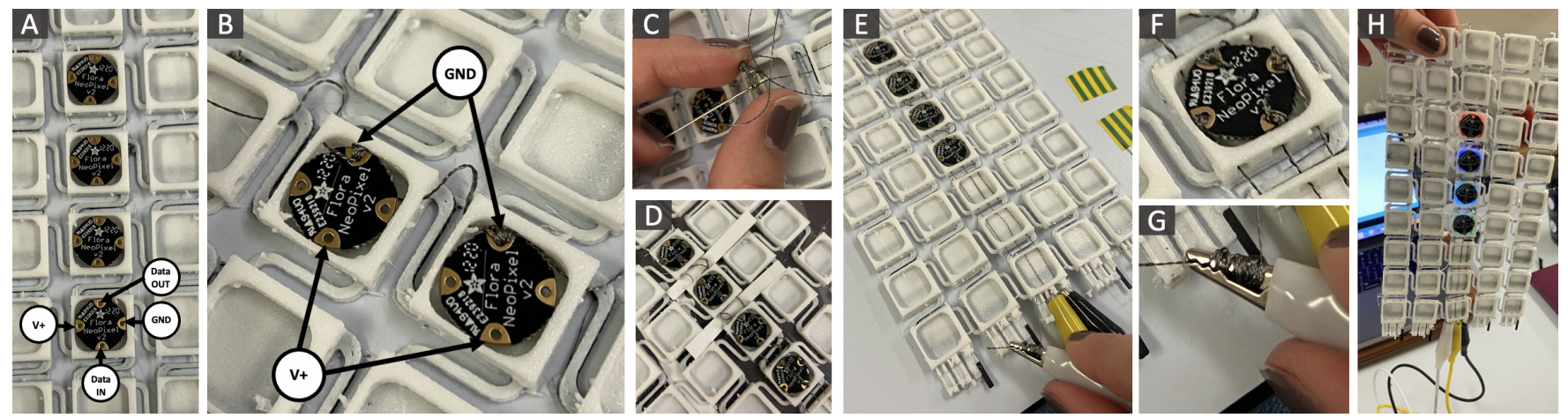

Figure 6: Step by step work-through for assembling a prototype using conductive thread and RGB LEDs [3]. Thus includes; placing LEDs into designated cavities (A); sewing conductive thread through LED chain (B-C); insulating thread with clear nail polish (D); and connecting the thread using crocodile clips (E-G) to secure it to a micro-controller for testing (H).

\subsection{Step 3: Assembly and Embedding Electronics}

To assemble the prototype we use conductive thread to connect the electronic components together. Figure 6 provides a step by step guide for assembly for a chain of four RGB LEDs. We begin by placing LEDs in the designated cavities making sure the text is the right way up (Figure 6A). We then secure conductive thread through all GND holes in the chain of LEDS (Figure 6B). We recommend threading the conductive thread through the holes at least 6 times before moving onto the next LED and repeat this process for $\mathrm{V}+$, Data IN, and Data OUT holes (Figure 6C). To insulate the conductive thread, first place white card dividers between each LED square and coat all conductive thread and LEDs with clear nail polish, spreading it generously (Figure 6D). Remove the white card dividers and wait for polish to dry for at least 2 hours. Once the polish is dry cut the thread between Data IN and Data OUT (Figure 6F) and wrap remainder of conductive thread around alligator clip (Figure 6E-G). Finally connect the alligator clips to a micro-controller board and test the LEDs work (Figure 6H).

We also used insulated electromagnetic wire $(0.5 \mathrm{~mm})$ on a smaller-scale prototype (tile dimensions $10 \times 10 \times 1.8 \mathrm{~mm}$ ) where the wire allows the device to hold its shape, unlike with thread. After repeated twisting and bending the wire does become fragile. To mitigate the risk of breaking we recommend burning off the insulating coating on the ends to expose the conductive copper beneath and applying solder directly on the wire to reinforce connections.

\subsection{Step 4: Circuity and Programming}

Figure 7 shows the circuit diagram details for setting up RGB LEDs for visualisation and touch-sensing using conductive filament for interaction. For touch sensing we use the Arduino Capacitive Sensing Library [7] in conjunction with a $60 \mathrm{OHM}$ resistor connected to the conductive filament. We use three pieces of conducive thread per row of LEDs, threaded into the channels in the tiles (Figure 3B). Capacitive readings from the conductive filament can detect when the device is worn by a user and when it is removed-e.g. contacting skin or lying on a table. The capacitive sensor library enables two or more Arduino or Adafruit Gemma pins to be a capacitive sensor that can detect the electrical capacitance of the human body. The sensor setup requires a medium to high value resistor (e.g. 100 kilohm - 50 megohm) and a piece of wire that is connected to the conductive filament on the end using copper tape or a crocodile clip (Figure 6G).

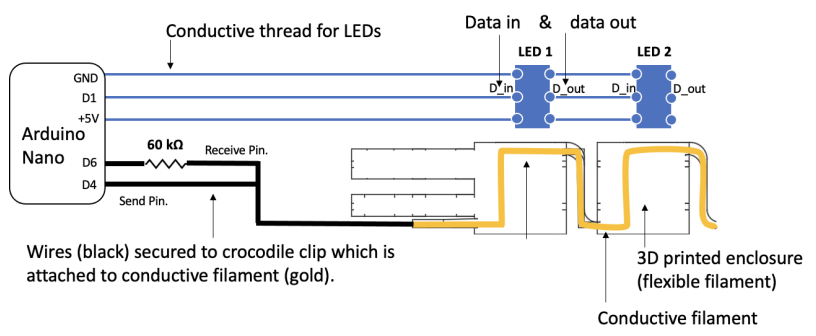

Figure 7: Circuit diagram for electronics, showing printed conductive tracks (yellow) and LEDs connected with conductive thread (blue).

\subsection{Step 5: Attachment}

Once the surface is fully assembled and functioning it can be attached to the body using double sided body tape [77], attached to clothing using pins, or other objects using conventional double sided tape or super glue. The device can also be easily secured onto the hand like a bracelet using clips or secured to other clothing/garments by directly sewing it on with thread.

\section{PROTOTYPE OVERVIEW}

Our prototype (Figure 8$)$ is a flexible 2D array of $8 \times 5$ tiles $(15 \times 15 \times 2$ $\mathrm{mm}$ and $5 \mathrm{~mm}$ apart), each connected with a curved link $(13 \times 1 \times 1$ $\mathrm{mm}$ ). To support rapid and iterative prototyping the design of the surface requires minimal print material, as the 3D models are thin $(2$ $\mathrm{mm}$ depth) and do not need support structures. The lack of support structures reduces printing time for our initial prototype (195x95x2 $\mathrm{mm})$ to $3 \mathrm{~h} 45 \mathrm{~min}$. A smaller-scale version $(145 \times 70 \times 1.8 \mathrm{~mm})$ takes $1 \mathrm{~h} 45 \mathrm{~min}$ to print. 


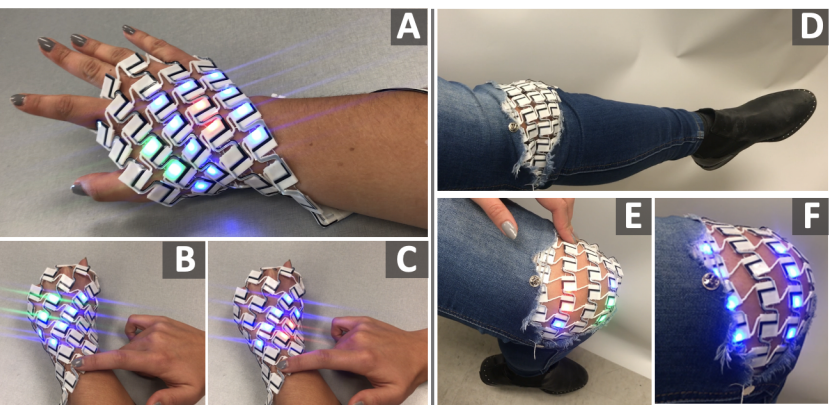

Figure 8: FlexiWear (195x95x2 mm) conforms to body shape and is easily worn on hand (A) with light (B) and hard (C) pressing, integrated with a pair of jeans $(D)$, pressing on bent knee (E) and stretched on bend knee (F).

\subsection{Interaction Capabilities}

We introduce interaction techniques supported by FlexiWear (Figure 2 and Figure 8), this includes; (1) capacitive touch sensing and (2) deformation detection on skin and stretching with movement. The interaction techniques below use the capacitive sensing library for Arduino [7].

4.1.1 Device Body Placement. We demonstrate interactions using two body placement examples. Figure $8 \mathrm{~A}-\mathrm{C}$ shows our prototype as a hand worn device that wraps, glove-like, around the wearer's hand. The hands and wrists are common locations for wearables [21]. Figure 8D-F shows our prototype integrated into a pair of jeans to detect knee bends. We use pins to attach the FlexiWear to the jeans. 3D printed devices like FlexiWear are robust and adapt to the body during movement. We describe these use cases below.

4.1.2 Touch Interactions. FlexiWear detects proximity of touch on tiles using capacitive thresholds set with the Arduino Uno. The conductive filament tracks (Figure 8) spans each row of tiles. The change in the capacitive sensing library's raw value output is a result from the deformation of the flexible filament and bringing the user's finger closer to the conductive filament (i.g., shortening the discharge cycle by sensing proximity of a conductor). We measure this change in output value for capacitive touch sensing to determine how close is a user's finger on the tile when they touch it. Figure $8 \mathrm{~A}$ shows green LEDs activated when a user lightly touches a tile. Figure $8 \mathrm{~B}$ shows red LEDs light up when a user touches the tile harder, as the capacitance reading increases above a set threshold. For interactive functionality, this capability to distinguish between light touches, where the finger is not so close to the conductive filament, and harder touches, where the finger is closer to the conductive filament, can be used as input control for common and uncommon tasks. For example, as a music control to pause a song a user can lightly touch a tile (e.g. common task). To skip a track (e.g. uncommon task), they press harder on the tile. This eliminates the need for both single and double touches.

4.1.3 Deformation Detection. Figure 8D-F shows FlexiWear adapting to bend and stretch with electronic circuits (i.e. a chain of LEDs) embedded within the prototype. Using capacitive input from the conductive material, we can detect human limb flexing and bending based on proximity of the skin to the conductive tiles when the sirface is stretched over a joint like the knee or elbow. For example, When the knee is extended, FlexiWear's surface is more loose fitted and contracted (Figure 8D), this is shown with the LEDs deactivated. When the knee is at 90 degrees, the surface is expanded and stretched across the knee, making the conductive filament have closer contact with the bare skin of the knee. Blue LEDs on the surface are activated (Figure 8F) to indicate that bending is detected. The system can simultaneously detect two separate inputs, one from the user's touch and a second from deformation with their body. By assigning one row of capacitive sensors a lower threshold for knee bending detection and another row a higher activation threshold for touch, the system can accommodate both. Figure 8E shows simultaneous knee bend and finger touch detection with a change in LED color (Figure 8D).

4.1.4 On-body Interaction. Using capacitive sensing we can detect when FlexiWear is not worn on the body. When the prototype is placed on the non-conductive table (e.g. wood), the capacitance reading is at its lowest. Once FlexiWear is placed on skin, there is an increase in capacitance from the conductive filament making close contact to the skin. This enables the device to distinguish between worn and not worn and could be used to control device modes, e.g. enter power saving mode when it is not being actively worn. Further explorations are needed for conductive surfaces (e.g. metal tables). These same capacitive sensors can be re-purposed for different uses through both software or hardware modifications (either by re-printing or with tools post-print). For example, we can detect knee bending based on the amount of contact the surface makes with the skin once it is stretched. The more the knee is bent, the greater the contact of the conductive material with the skin, resulting in a higher capacitance input reading. 3D printed flexible surfaces can also stretch based on the interconnected link designs as demonstrated by Schumacher et al. [71]. Generic stretch sensors can also be incorporated to measure stretch when no skin contact is made with the surface.

\section{DISCUSSION}

Here we discuss the practical advantages of our design and fabrication approach, elaborating on design considerations, expand on future work, as well as highlight current limitations and how we aim to address them.

\subsection{Advantages of Approach}

5.1.1 Tile Design. To create the FlexiWear prototype, a user only needs to create one tile and one link in a CAD environment (e.g. one square with a link). The set is then replicated (using the copy and move function available in most CAD environments) to form an array of tiles that are linked together. After, tiles and linkages can be modified and connected in different configurations to change the overall shape and properties of the device. We believe that this replication focused $\mathrm{CAD}$ design approach reduces the complexity of the design process during prototyping. Additionally, once the user is satisfied with their digital design, they 3D print the surface, insert post-print components, and make a connection between the conductive filament to an Arduino using an Alligator clip or silver paste. 
5.1.2 Reduced Complex Assembly Requirements. Recent work in e-textiles and wearables consist of multilayer fabrication processes that require extensive manual assembly and advanced laser micromachining that is capable of processing thin metal films [12, 25, 47]. Similarly, stretchable thin film wearables consist of multi-layer structures [84] that require handling chemicals such as phosphor and silicon-based organic polymers. These approaches offer increased technical complexity due to their multi-layer structures. To reduce technical complexity and support a more accessible design and development method, our fabrication approach consists of two primary elements that are integrated into a single-layer wearable surface: (1) The multi-material surface layer that integrates flexible and conductive filament during the $3 \mathrm{D}$ printing process to support input capabilities without the need for additional electronic sensors. (2) The additional layer of electronic components, like LEDs, that are inserted within the $3 \mathrm{D}$ printed stretchable surface for output visualizations. These components can be embedded in the flexible surface. Though our simple device prototype consisting of two elements, conductive track for capacitive sensing and an array of LEDs, there are still manual assembly requirements. Specifically, as each LED has to be manually inserted into a tile and soldered there is a scalability trade-off, where more LEDs increase the manual assembly requirements.

5.1.3 Rapid Fabrication and Replicability. By utilizing multi-material 3D printing, with integrated conductive and flexible filaments, we can reduce the cost of time and provide fabricators with broad control of the prototyping process. With this method is it possible to mimic the deformable properties of e-textiles, dictate the rigidity and stretch of all or some parts of the prototype, add and alter capacitive circuits, and modify the printed substrate to support different components for embedded interactive features. We highlight the rapid nature of fabricating FlexiWear style prototypes: where a $195 \times 95 \times 2 \mathrm{~mm}$ surface takes $3 \mathrm{~h} 45 \mathrm{~min}$ to $3 \mathrm{D}$ print. A smaller-scale version (145x70x1.8 mm) takes $1 \mathrm{~h} 45 \mathrm{~min}$ to print. Inserting LEDs in the surface and wiring took an additional $30 \mathrm{~min}$. Fabricating a fully functioning wearable prototype using our approach took, in total, 4 hours and $30 \mathrm{~min}$. To demonstrate the potential for replicability of our fabrication process we built 25 prototypes in total, 21 of which have been used as toolkits for a Masters' remote workshop on prototyping wearable technologies. Each prototype packed and sent to students homes. Each student was able to be set-up and assembled the prototype during a Zoom workshop without any technical failure. We believe this demonstrate the robust and replicable nature of our prototypes.

\subsection{Design Considerations}

5.2.1 Stretch Factor and Durability. When 3D modeling flexible links and tiles, designers must take scale into consideration. A thicker link ensures a more robust connection and less chance of breakage when stretched with greater force but limits overall malleability. The stretching capabilities of FlexiWear also depend on where conductive filament is located. Conductive PLA can bend when encased in flexible material and printed in a single layer (e.g. $0.2 \mathrm{~mm}$ ). The long-term robustness of this adaptation is unexplored but sufficient for iterative prototyping and testing.
To increase stretch-ability, the link length can be increased, or its shape changed. The stretching factor works like a 2D spring coil, where the number of "coils" and their length affect the stretching. For example, if the link has one coil with length of $10 \mathrm{~mm}$ between two tiles, then the stretched gap between those two tiles will be $10 \mathrm{~mm}$. If the link has two coils with length of $10 \mathrm{~mm}$ then the gap will be $20 \mathrm{~mm}$. Using $10 \mathrm{~mm}$ links, a 5-tile array with 4 links can extend up to $40 \mathrm{~mm}$ from its non-deformed size. At rest, a maximum gap of $2 \mathrm{~mm}$ between each tile should be maintained to ensure the tile array behaves as a singular surface. Based on our link design explorations, we recommend curved links over right-angles, particularly for durability. Figure 5C shows the final link design to be curved where it attaches to the tiles. This curvature reduces the risk of tearing the link. The design consideration for link design promotes durability whilst the surface is stretching.

5.2.2 Sensing Capabilities. Additional design questions on sensing capabilities that support future work have also emerged. Precariously, how long can the conductive tracks can be and still be able to detect touch at the end? The conductive filament resistance can be decreased by making the printed lines thicker or wider, however this will be more difficult to bend or deform the surface. There are additional design considerations on the geometry of the conductive lines for optimal sensing and detection. The choice of the resistance between the pulse pin and reading pin can also affect the interactive capabilities of the surface. The physical setup includes a medium to high value (100 kilohm - 50 megohm) resistor between the send pin and the receive (sensor) pin. One trade-off with larger resistors is that the sensor's increased sensitivity means that it is slower. Also, if the surface is placed on exposed metal, it is possible that the send pin will never be able to force a change in the receive sensor pin, and the sensor will timeout.

\subsection{Future Work}

In future work we aim integrate a wider range of sensors and components to support input and output capabilities, such as Light Dependent Resistors (LDRs) and actuators like muscle wires/shapememory alloys (SMAs). We also aim to explore a range of body placements and experiment with tile designs and links. Currently, the 3D printed FlexiWear devices are limited by the size of the print bed. To create larger surfaces, we will explore patchwork designs with conductive interlinks. Designs that fill gaps created during deformation are important, currently gaps can catch on clothing or other obstructions.

In terms of design accessibility and democratizing technology [76], we hope our approach will be adopted by Maker culture. By publishing our 3D model online in open source modeling libraries, we aim to expand the reach of 3D printed wearables, beyond etextiles, to hobbyists and designers. Through making our models open-source (e.g. Thingiverse.com) we hope to facilitate designing prototypes and bespoke wearables.

In future work, we also aim to integrate self-actuation capabilities as detailed by Roudaut et al. [67] or incorporate modular origami robots [9] for actuation. Finally, we envision a range of quantitative and qualitative user studies to evaluate how participants prefer interacting with our prototype and use these findings to inform usability paradigms for deformable devices and support 
the exploration for potential adoption of these devices. We aim to develop a range of application examples that utilize the deformable material properties and interactions supported by FlexiWear. In the area of robotics we envision the surfaces as robotic skins that can stretch and flex with the joint movement of a robotic arm. Int he area of digital health, we also aim to use FlexiWear as devices used for working-out to potentially improve the people's awareness of their bodies while exercising. Different exercises require different on-body sensing, such as push-up (device on the elbows), or squats and dead lifts (on the knees).

\subsection{Limitations}

In terms of input and output electronics, we acknowledge that capacitive sensing and LEDs have been extensively used in HCI. For this work, these components were used to demonstrate the generalizability and accessibility of this prototyping method. Though flexible printed electronics offer better conductors (silver nanoparticles) over 3D printed conductors (graphene-based filament), those techniques are not as affordable or accessible. Additionally, we have limited validation for the usability of our approach as there is no user study or initial user feedback provided which would allow to gauge whether the approach is easy-to-use. For our next step, we aim to demonstrate the utility of our approach with a qualitative user study, following a similarly methodology for toolkit user validation as pre-existing work [37].

5.4.1 Post-Printing Assembly Requirements. The post processing does require some assembly to be done by hand (e.g. securing the conductive thread to the LEDs and placing LEDs within the 3D printed enclosure). This does partly lose some of the advantages that automated 3D printing brings, nevertheless connecting the LEDs and placing them into an enclosure is a menial task in comparison to the multi-layer assembly processes fabrication techniques such as ElectroDermis [47] or Stretchis [84] offer. Additionally, there is potential for a user to develop favourable attraction towards a prototype that they assemble manually for themselves. Nevertheless, for future work, our method can be further automated by adopting techniques from latest work in acoustic fabrication with automated contactless pick and place of electronic components [5] to further reduce assembly requirements.

5.4.2 Circuit Limitations. Currently realized structure only allows for row-based sensing and activation of the LEDs. This is because our demo has no electronic connections between rows and all capacitors are in a series in one row. This design decision was predominantly intended to reduce the complexity of the 3D printed structure. A full matrix like configuration would be a more desirable structure design for individual tile sensing capabilities. For more efficient capacitive touch sensing a structured matrices [4] design would be more desirable in future iterations of the prototype. The LED control for our demo prototype (Figure 8) is also row by row control which is a limitation. Using PCB RBG LEDs with an additional input data pin can provide individual control to each LED as illustrated in Figure 7.

5.4.3 Technical Clarifications and Scalability. The current sensing capabilities of our prototype requires further testing. A large body of work within Human Computer Interaction explores these capacitive sensing techniques and highlights current challenges and limitations with simplistic circuitry and limited location based tracking [24] that capacitive sensing offers. Currently, we promote this method for early prototyping and our initial bend interaction tests with capacitive sensing provide enough accuracy for rough input sensing. Capacitive sensing can be prone to noise and can potentially show different characteristics when the prototype is on the skin or on cloth. We manually configured the input thresholds for each prototype when placed on the skin or on cloth. In future work we also aim to integrate other forms of sensing for input into our prototypes. User-aware sensing could include an accelerometer to provide a more accurate input when the device is placed on a conductive surface such as a metal table. The system can potentially be improved as currently each cell requires a separate control system if not daisy chained in sequence. Multiplexing can support multiple analog and digital signals over a shared medium. Alternatively, as mentioned above, a full matrix structure design with both column and row connections can also provide a more scalable design.

5.4.4 Flexibility and Stretching. The electrical wires, connection, and architecture of the tiles create an anisotropic flexibility with limited stretching. This means that the stretching ability is limited along the length of the lines (Figure 2C). This limited stretching can be overcome by adding more lines to each link, much like a spring has more coils to increase the length of stretch.

\section{CONCLUSION}

This work explores how multi-material 3D printing can support the fabrication of deformable wearables with interaction and visualization capabilities. Our multi-material 3D printing approach focuses on an affordable and accessible fabrication process that does not require highly technical or expensive fabrication tools for prototyping deformable wearables. Through our FlexiWear prototypes, we demonstrated that this approach is capable of fabricating thin deformable surfaces that are flexible, foldable, and stretchable. We believe that the wearable electronics community can benefit from utilizing multi-material 3D printing for fabricating wearables. Additionally, we also aim to introduce even novice makers and hobbyists to developing their own stretchable and flexible wearables using 3D printers.

Our core motivation is to provide the research and design communities with an alternate rapid and accessible means of fabricating deformable wearable prototypes that can be worn on skin (Figure $2 \mathrm{~A}$ ) or integrated in garments (Figure 2C). We demonstrate this by presenting a rapid and customizable fabrication approach that can support a range of wearable forms and functions, is easily augmented with off-the shelf components, and modifiable with standard tools. By designing in digital environments, we support fast iterations (print times and modifications), inexpensive and easily sourced materials, which speed up assembly with commonly available tools.

To summarize, the key contribution of this work is our unique tile and surface design that supports low-cost fabrication approach for developing interactive deformable wearable prototypes using multi-material 3D printing. Our deformable wearables (FlexiWear) adapt to various body shapes, joints, and can be integrated in garments. Specifically, with minimal time and assembly requirements. 
We highlight how FlexiWear demonstrates that multi-material 3D printing offers rapid fabrication of prototypes that can be used to test a wide range of wearable and mobile concepts.

\section{ACKNOWLEDGMENTS}

Big thank you to Alfrancis Guerrero for helping with the 3D printing. This work was supported and funded by the National Sciences and Engineering Research Council of Canada (NSERC) through a Discovery grant (2017-06300). The research was also supported by Amazon Web Services in the Oxford-Singapore Human-Machine Collaboration Programme.

\section{REFERENCES}

[1] Adafruit. 2021. Adafruit Gemma v2. Retrieved Feb 6, 2021 from https://www. adafruit.com/product/1222

[2] Adafruit. 2021. Adafruit LED Sequins. Retrieved Feb 6, 2021 from https //www.adafruit.com/product/1757

[3] Adafruit. 2021. Adafruit NeoPixel LED. Retrieved Feb 6, 2021 from https: //www.adafruit.com/product/1260

[4] Humza Akhtar and Ramakrishna Kakarala. 2015. Efficient capacitive touch sensing using structured matrices. In Computational Imaging XIII, Vol. 9401. International Society for Optics and Photonics, $94010 \mathrm{O}$

[5] Marco AB Andrade, Tiago S Ramos, Julio C Adamowski, and Asier Marzo. 2020. Contactless pick-and-place of millimetric objects using inverted near-field acoustic levitation. Applied Physics Letters 116, 5 (2020), 054104.

[6] Arduino. 2021. Arduino Nano. Retrieved Feb 6, 2021 from https://store.arduino. cc/arduino-nano

[7] Paul Badger. 2018. Capacitive Sensing Library. Retrieved Sept 10, 2020 from https://playground.arduino.cc/Main/CapacitiveSensor/

[8] Patrick Baudisch, Stefanie Mueller, et al. 2017. Personal fabrication. Foundations and Trends ${ }^{\circledR}$ in Human-Computer Interaction 10, 3-4 (2017), 165-293.

[9] Christoph H Belke and Jamie Paik. 2017. Mori: a modular origami robot. IEEE/ASME Transactions on Mechatronics 22, 5 (2017), 2153-2164.

[10] Leah Buechley, Mike Eisenberg, Jaime Catchen, and Ali Crockett. 2008. The LilyPad Arduino: using computational textiles to investigate engagement, aesthetics, and diversity in computer science education. In Proceedings of the SIGCHI conference on Human factors in computing systems. 423-432.

[11] Jesse Burstyn, Paul Strohmeier, and Roel Vertegaal. 2015. DisplaySkin: Exploring pose-aware displays on a flexible electrophoretic wristband. In Proceedings of the Ninth International Conference on Tangible, Embedded, and Embodied Interaction. 165-172.

[12] Lina M Castano and Alison B Flatau. 2014. Smart fabric sensors and e-textile technologies: a review. Smart Materials and structures 23, 5 (2014), 053001.

[13] Victor Cheung, Alex Keith Eady, and Audrey Girouard. 2017. Exploring eyes-free interaction with wrist-worn deformable materials. In Proceedings of the Eleventh International Conference on Tangible, Embedded, and Embodied Interaction. 521528.

[14] William Cheung and Sudip Vhaduri. 2020. Context-Dependent Implicit Authentication for Wearable Device User. arXiv preprint arXiv:2008.12145 (2020).

[15] Kenneth E Evans and Andrew Alderson. 2000. Auxetic materials: functional materials and structures from lateral thinking! Advanced materials 12, 9 (2000), 617-628.

[16] Aluna Everitt and Jason Alexander. 2017. PolySurface: a design approach for rapid prototyping of shape-changing displays using semi-solid surfaces. In Proceedings of the 2017 Conference on Designing Interactive Systems. 1283-1294.

[17] Aluna Everitt and Jason Alexander. 2019. 3D Printed Deformable Surfaces for Shape-Changing Displays. Frontiers in Robotics and AI 6 (2019), 80.

[18] Fennerdrives.com. 2021. Cheetah ${ }^{\mathrm{TM}}$ Flexible 3D Printing Filament 95A TPU - Industrial Strength Material. Retrieved Feb 6, 2021 from https://www.fennerdrives.com/product-lines/_/ninjatek/cheetah?utm_ source=ninjatek.com\&utm medium $=$ referral\&utm campaign=button

[19] GHEORGHE Florea, RADU Dobrescu, D Popescu, and MATEI Dobrescu. 2013. Wearable system for heat stress monitoring in firefighting applications. In Recent Advances in Computer Science and Networking: Proceedings of the 2nd International Conference on Information Technology and Computer Networks. 129-134.

[20] Minoru Fujimoto, Fujita Naotaka, Tsutomu Terada, and Masahiko Tsukamoto. 2011. Lighting choreographer: an LED control system for dance performances. In Proceedings of the 13th international conference on Ubiquitous computing. 613-614.

[21] Francine Gemperle, Chris Kasabach, John Stivoric, Malcolm Bauer, and Richard Martin. 1998. Design for wearability. In digest of papers. Second international symposium on wearable computers (cat. No. 98EX215). IEEE, 116-122.

[22] Nan-Wei Gong, Jürgen Steimle, Simon Olberding, Steve Hodges, Nicholas Edward Gillian, Yoshihiro Kawahara, and Joseph A Paradiso. 2014. PrintSense: a versatile sensing technique to support multimodal flexible surface interaction. In Proceedings of the SIGCHI Conference on Human Factors in Computing Systems. $1407-1410$.

[23] Daniel Groeger and Jürgen Steimle. 2019. LASEC: Instant Fabrication of Stretchable Circuits Using a Laser Cutter. In Proceedings of the 2019 CHI Conference on Human Factors in Computing Systems. 1-14.

[24] Tobias Grosse-Puppendahl, Christian Holz, Gabe Cohn, Raphael Wimmer, Oskar Bechtold, Steve Hodges, Matthew S Reynolds, and Joshua R Smith. 2017. Finding common ground: A survey of capacitive sensing in human-computer interaction. In Proceedings of the 2017 CHI Conference on Human Factors in Computing Systems. 3293-3315.

[25] Nur Al-huda Hamdan, Simon Voelker, and Jan Borchers. 2018. Sketch\&stitch: Interactive embroidery for e-textiles. In Proceedings of the 2018 CHI Conference on Human Factors in Computing Systems. 1-13.

[26] Sunao Hashimoto, Ryohei Suzuki, Youichi Kamiyama, Masahiko Inami, and Takeo Igarashi. 2013. LightCloth: senseable illuminating optical fiber cloth for creating interactive surfaces. In Proceedings of the SIGCHI Conference on Human Factors in Computing Systems. 603-606.

[27] Liang He, Huaishu Peng, Joshua Land, Mark D Fuge, and Jon E Froehlich. 2017. Designing 3D-printed deformation behaviors using spring-based structures: an initial investigation. In Adjunct Publication of the 30th Annual ACM Symposium on User Interface Software and Technology. 151-153.

[28] Paul Holleis, Albrecht Schmidt, Susanna Paasovaara, Arto Puikkonen, and Jonna Häkkilä. 2008. Evaluating capacitive touch input on clothes. In Proceedings of the 10th international conference on Human computer interaction with mobile devices and services. $81-90$.

[29] Scott E Hudson. 2014. Printing teddy bears: a technique for 3D printing of soft interactive objects. In Proceedings of the SIGCHI Conference on Human Factors in Computing Systems. 459-468.

[30] Kohei Ikeda, Naoya Koizumi, and Takeshi Naemura. 2017. FunCushion: fabricating functional cushion interfaces with fluorescent-pattern displays. In International Conference on Advances in Computer Entertainment. Springer, 470-487.

[31] Margot Jacobs and Linda Worbin. 2005. Reach: dynamic textile patterns for communication and social expression. In CHI'05 Extended Abstracts on Human Factors in Computing Systems. 1493-1496.

[32] Walther Jensen, Ashley Colley, Jonna Häkkilä, Carlos Pinheiro, and Markus Löchtefeld. 2019. TransPrint: A method for fabricating flexible transparent freeform displays. Advances in Human-Computer Interaction 2019 (2019).

[33] Lee Jones, Sara Nabil, Amanda McLeod, and Audrey Girouard. 2020. Wearable Bits: scaffolding creativity with a prototyping toolkit for wearable e-textiles. In Proceedings of the Fourteenth International Conference on Tangible, Embedded, and Embodied Interaction. 165-177.

[34] Viirj Kan, Katsuya Fujii, Judith Amores, Chang Long Zhu Jin, Pattie Maes, and Hiroshi Ishii. 2015. Social textiles: Social affordances and icebreaking interactions through wearable social messaging. In Proceedings of the Ninth International Conference on Tangible, Embedded, and Embodied Interaction. 619-624.

[35] Hsin-Liu Kao, Christian Holz, Asta Roseway, Andres Calvo, and Chris Schmandt. 2016. DuoSkin: rapidly prototyping on-skin user interfaces using skin-friendly materials. In Proceedings of the 2016 ACM International Symposium on Wearable Computers. 16-23.

[36] Johan Kildal. 2012. Interacting with deformable user interfaces: effect of material stiffness and type of deformation gesture. In International Conference on Haptic and Audio Interaction Design. Springer, 71-80.

[37] Hyunyoung Kim, Aluna Everitt, Carlos Tejada, Mengyu Zhong, and Daniel Ashbrook. 2021. MorpheesPlug: A Toolkit for Prototyping Shape-Changing Interfaces. In Proceedings of the 2021 CHI Conference on Human Factors in Computing Systems. $1-13$.

[38] Jaemin Kim, Jongsu Lee, Donghee Son, Moon Kee Choi, and Dae-Hyeong Kim. 2016. Deformable devices with integrated functional nanomaterials for wearable electronics. Nano Convergence 3, 1 (2016), 1-13.

[39] William Kylberg, Fernando Araujo De Castro, Peter Chabrecek, Uriel Sonderegger, Bryan Tsu-Te Chu, Frank Nüesch, and Roland Hany. 2011. Woven electrodes for flexible organic photovoltaic cells. Advanced Materials 23, 8 (2011), 1015-1019.

[40] Isabel Leber and Natividad Martínez Madrid. 2018. WearIT-a rapid prototyping platform for wearables. In International Conference on Bioinformatics and Biomedical Engineering. Springer, 335-346.

[41] Jeongwoo Lee, Ho-Chan Kim, Jae-Won Choi, and In Hwan Lee. 2017. A review on $3 \mathrm{D}$ printed smart devices for $4 \mathrm{D}$ printing. International fournal of Precision Engineering and Manufacturing-Green Technology 4, 3 (2017), 373-383.

[42] Seulki Lee, Binhee Kim, and Hoi-Jun Yoo. 2009. Planar fashionable circuit board technology and its applications. Journal of Semiconductor Technology and Science 9, 3 (2009), 174-180.

[43] Jessica Lo and Audrey Girouard. 2014. Fabricating bendy: Design and development of deformable prototypes. IEEE Pervasive Computing 13, 3 (2014), 40-46.

[44] Joanne Lo, Doris Jung Lin Lee, Nathan Wong, David Bui, and Eric Paulos. 2016. Skintillates: Designing and creating epidermal interactions. In Proceedings of the 2016 ACM Conference on Designing Interactive Systems. 853-864. 
[45] Kent Lyons, David Nguyen, Daniel Ashbrook, and Sean White. 2012. Facet: a multi-segment wrist worn system. In Proceedings of the 25th annual ACM symposium on User interface software and technology. 123-130.

[46] Diana Marculescu, Radu Marculescu, Nicholas H Zamora, Phillip Stanley-Marbell, Pradeep K Khosla, Sungmee Park, Sundaresan Jayaraman, Stefan Jung, Christl Lauterbach, Werner Weber, et al. 2003. Electronic textiles: A platform for pervasive computing. Proc. IEEE 91, 12 (2003), 1995-2018.

[47] Eric Markvicka, Guanyun Wang, Yi-Chin Lee, Gierad Laput, Carmel Majidi, and Lining Yao. 2019. ElectroDermis: Fully Untethered, Stretchable, and HighlyCustomizable Electronic Bandages. In Proceedings of the 2019 CHI Conference on Human Factors in Computing Systems. 1-10.

[48] James McCann, Lea Albaugh, Vidya Narayanan, April Grow, Wojciech Matusik, Jennifer Mankoff, and Jessica Hodgins. 2016. A compiler for 3D machine knitting. ACM Transactions on Graphics (TOG) 35, 4 (2016), 1-11.

[49] David A Mellis, Leah Buechley, Mitchel Resnick, and Björn Hartmann. 2016. Engaging amateurs in the design, fabrication, and assembly of electronic devices. In Proceedings of the 2016 ACM Conference on Designing Interactive Systems. 12701281.

[50] C Mota. 2011. The rise of personal fabrication In: Proceedings of the 8th ACM conference on Creativity and cognition.

[51] Troy Nachtigall. 2017. EVA Moccasin: creating a research archetype to explore shoe use. In Proceedings of the 2017 ACM International Symposium on Wearable Computers. 197-202.

[52] Troy Nachtigall, Daniel Tetteroo, and Panos Markopoulos. 2018. A five-year review of methods, purposes and domains of the international symposium on wearable computing. In Proceedings of the 2018 ACM International Symposium on Wearable Computers. 48-55.

[53] Steven Nagels, Raf Ramakers, Kris Luyten, and Wim Deferme. 2018. Silicone devices: A scalable DIY approach for fabricating self-contained multi-layered soft circuits using microfluidics. In Proceedings of the 2018 CHI Conference on Human Factors in Computing Systems. 1-13.

[54] Grace Ngai, Stephen CF Chan, Joey CY Cheung, and Winnie WY Lau. 2009 The TeeBoard: an education-friendly construction platform for e-textiles and wearable computing. In Proceedings of the SIGCHI Conference on Human Factors in Computing Systems. 249-258.

[55] Aditya Shekhar Nittala, Anusha Withana, Narjes Pourjafarian, and Jürgen Steimle. 2018. Multi-touch skin: A thin and flexible multi-touch sensor for on-skin input In Proceedings of the 2018 CHI Conference on Human Factors in Computing Systems. $1-12$.

[56] Simon Olberding, Kian Peen Yeo, Suranga Nanayakkara, and Jurgen Steimle. 2013 AugmentedForearm: exploring the design space of a display-enhanced forearm. In Proceedings of the 4th Augmented Human International Conference. 9-12.

[57] Jifei Ou, Daniel Oran, Don Derek Haddad, Joseph Paradiso, and Hiroshi Ishii 2019. SensorKnit: architecting textile sensors with machine knitting. 3D Printing and Additive Manufacturing 6, 1 (2019), 1-11.

[58] Antti Oulasvirta and Gregory D Abowd. 2016. User interface design in the 21st century. Computer 49, 7 (2016), 11-13.

[59] Roshan Lalintha Peiris, Mili John Tharakan, Adrian David Cheok, and Owen Noel Newton. 2011. AmbiKraf: a ubiquitous non-emissive color changing fabric display. In Proceedings of the 15th International Academic MindTrek Conference: Envisioning Future Media Environments. 320-322.

[60] Huaishu Peng, Jennifer Mankoff, Scott E Hudson, and James McCann. 2015. A layered fabric 3D printer for soft interactive objects. In Proceedings of the 33rd Annual ACM Conference on Human Factors in Computing Systems. 1789-1798.

[61] Ivan Poupyrev, Nan-Wei Gong, Shiho Fukuhara, Mustafa Emre Karagozler Carsten Schwesig, and Karen E Robinson. 2016. Project Jacquard: interactive digital textiles at scale. In Proceedings of the 2016 CHI Conference on Human Factors in Computing Systems. 4216-4227.

[62] Proto-pasta.com. 2021. Electrically Conductive Composite PLA. Retrieved Feb 6, 2021 from https://www.proto-pasta.com/products/conductive-pla?variant= 1265211484

[63] Isabel PS Qamar, Rainer Groh, David Holman, and Anne Roudaut. 2018. HCI meets material science: A literature review of morphing materials for the design of shape-changing interfaces. In Proceedings of the 2018 CHI Conference on Human Factors in Computing Systems. 1-23.

[64] Raf Ramakers, Johannes Schöning, and Kris Luyten. 2014. Paddle: highly deformable mobile devices with physical controls. In Proceedings of the SIGCHI Conference on Human Factors in Computing Systems. 2569-2578.

[65] Gregory B Raupp, Shawn M O'Rourke, Curt Moyer, Barry P O'Brien, Scott K Ageno, Douglas E Loy, Edward J Bawolek, David R Allee, Sameer M Venugopal, Jann Kaminski, et al. 2007. Low-temperature amorphous-silicon backplane technology development for flexible displays in a manufacturing pilot-line environment. Fournal of the Society for Information Display 15, 7 (2007), 445-454.

[66] Michael L Rivera, Melissa Moukperian, Daniel Ashbrook, Jennifer Mankoff, and Scott E Hudson. 2017. Stretching the bounds of 3D printing with embedded textiles. In Proceedings of the 2017 CHI Conference on Human Factors in Computing Systems. 497-508.
[67] Anne Roudaut, Abhijit Karnik, Markus Löchtefeld, and Sriram Subramanian. 2013. Morphees: toward high" shape resolution" in self-actuated flexible mobile devices. In Proceedings of the SIGCHI Conference on Human Factors in Computing Systems. 593-602.

[68] Valkyrie Savage, Ryan Schmidt, Tovi Grossman, George Fitzmaurice, and Björn Hartmann. 2014. A series of tubes: adding interactivity to 3D prints using internal pipes. In Proceedings of the 27th annual ACM symposium on User interface software and technology. 3-12.

[69] Martin Schmitz, Mohammadreza Khalilbeigi, Matthias Balwierz, Roman Lissermann, Max Mühlhäuser, and Jürgen Steimle. 2015. Capricate: A fabrication pipeline to design and 3D print capacitive touch sensors for interactive objects. In Proceedings of the 28th Annual ACM Symposium on User Interface Software \& Technology. 253-258.

[70] Martin Schmitz, Jürgen Steimle, Jochen Huber, Niloofar Dezfuli, and Max Mühlhäuser. 2017. Flexibles: deformation-aware 3D-printed tangibles for capacitive touchscreens. In Proceedings of the 2017 CHI Conference on Human Factors in Computing Systems. 1001-1014.

[71] Christian Schumacher, Steve Marschner, Markus Gross, and Bernhard Thomaszewski. 2018. Mechanical characterization of structured sheet materials. ACM Transactions on Graphics (TOG) 37, 4 (2018), 1-15.

[72] Carsten Schwesig, Ivan Poupyrev, and Eijiro Mori. 2004. Gummi: a bendable computer. In Proceedings of the SIGCHI conference on Human factors in computing systems. 263-270.

[73] Teddy Seyed, Xing-Dong Yang, and Daniel Vogel. 2016. Doppio: A Reconfigurable Dual-Face Smartwatch for Tangible Interaction. In Proceedings of the $2016 \mathrm{CHI}$ Conference on Human Factors in Computing Systems. 4675-4686.

[74] Yuta Sugiura, Gota Kakehi, Anusha Withana, Calista Lee, Daisuke Sakamoto, Maki Sugimoto, Masahiko Inami, and Takeo Igarashi. 2011. Detecting shape deformation of soft objects using directional photoreflectivity measurement. In Proceedings of the 24th annual ACM symposium on User interface software and technology. 509-516.

[75] Ruojia Sun, Ryosuke Onose, Margaret Dunne, Andrea Ling, Amanda Denham, and Hsin-Liu Kao. 2020. Weaving a Second Skin: Exploring Opportunities for Crafting On-Skin Interfaces Through Weaving. In Proceedings of the 2020 ACM Designing Interactive Systems Conference. 365-377.

[76] Joshua G Tanenbaum, Amanda M Williams, Audrey Desjardins, and Karen Tanenbaum. 2013. Democratizing technology: pleasure, utility and expressiveness in DIY and maker practice. In Proceedings of the SIGCHI Conference on Human Factors in Computing Systems. 2603-2612.

[77] LocoTime Body Tape. 2021. LocoTime - Double Sided Fashion Body Tape for Clothing. Retrieved Feb 6, 2021 from https://www.amazon.co.uk/FashionBody-Clothing-Lingerie-Dispenser/dp/B073PYR2VK/ref=pd_lpo_79_t_0/2606401149-5616508?_encoding=UTF8\&pd_rd_i=B073PYXG3D\&pd_rd_r= 381e1433-bdb9-4468-b5a7-c4405490f0e8\&pd_rd w=XY5Ox\&pd_rd wg=bSBa4\&pf_rd_p=da0677f5-a47b-4543-8b54-10be576b8f26\&pf_rd_r= PRGF5A0MACHZ2A1TGC8J\&psc $=1 \&$ refRID=PRGF5A0MACHZ2A1TGC8J

[78] Aneesh P Tarun, Byron Lahey, Audrey Girouard, Winslow Burleson, and Roel Vertegaal. 2011. Snaplet: using body shape to inform function in mobile flexible display devices. In CHI'11 Extended Abstracts on Human Factors in Computing Systems. 329-334.

[79] Ryan L Truby, Michael Wehner, Abigail K Grosskopf, Daniel M Vogt, Sebastien GM Uzel, Robert J Wood, and Jennifer A Lewis. 2018. Soft somatosensitive actuators via embedded 3D printing. Advanced Materials 30, 15 (2018), 1706383.

[80] Kevin Vlack, Terukazu Mizota, Naoki Kawakami, Kazuto Kamiyama, Hiroyuki Kajimoto, and Susumu Tachi. 2005. GelForce: a vision-based traction field computer interface. In CHI'05 extended abstracts on Human factors in computing systems. $1154-1155$.

[81] Ulrich Von Zadow, Wolfgang Büschel, Ricardo Langner, and Raimund Dachselt. 2014. Sleed: Using a sleeve display to interact with touch-sensitive display walls. In Proceedings of the Ninth ACM International Conference on Interactive Tabletops and Surfaces. 129-138.

[82] Martin Weigel, Tong Lu, Gilles Bailly, Antti Oulasvirta, Carmel Majidi, and Jürgen Steimle. 2015. Iskin: flexible, stretchable and visually customizable on-body touch sensors for mobile computing. In Proceedings of the 33rd Annual ACM Conference on Human Factors in Computing Systems. 2991-3000.

[83] Martin Weigel, Aditya Shekhar Nittala, Alex Olwal, and Jürgen Steimle. 2017. Skinmarks: Enabling interactions on body landmarks using conformal skin electronics. In Proceedings of the 2017 CHI Conference on Human Factors in Computing Systems. 3095-3105.

[84] Michael Wessely, Theophanis Tsandilas, and Wendy E Mackay. 2016. Stretchis: Fabricating highly stretchable user interfaces. In Proceedings of the 29th Annual Symposium on User Interface Software and Technology. 697-704.

[85] Kaufui V Wong and Aldo Hernandez. 2012. A review of additive manufacturing. International scholarly research notices 2012 (2012).

[86] Te-Yen Wu, Shutong Qi, Junchi Chen, MuJie Shang, Jun Gong, Teddy Seyed, and Xing-Dong Yang. 2020. Fabriccio: Touchless Gestural Input on Interactive Fabrics. In Proceedings of the $2020 \mathrm{CHI}$ Conference on Human Factors in Computing Systems. $1-14$. 